\title{
Spatial Stress and Strain Distributions of Viscoelastic Layers in Oscillatory Shear
}

\author{
Brandon S. Lindley ${ }^{a}{ }^{\star}$, M. Gregory Forest ${ }^{\mathrm{b}}$, Breannan D. Smith $^{\mathrm{c}}$, Sorin M. Mitran ${ }^{\mathrm{b}}$, and David \\ B. Hill ${ }^{\mathrm{d}}$ \\ aInterdisciplinary Mathematics Institute, Department of Mathematics, University of South Carolina, \\ Columbia, 29208 \\ bDepartment of Mathematics, University of North Carolina, Chapel Hill, 27599-3250 \\ 'Department of Computer Science, Columbia University, New York, 10027-7003 \\ ${ }^{\mathrm{d} C y s t i c}$ Fibrosis Center, University of North Carolina, Chapel Hill, 27599-3250
}

\begin{abstract}
One of the standard experimental probes of a viscoelastic material is to measure the response of a layer trapped between parallel surfaces, imposing either periodic stress or strain at one boundary and measuring the other. The relative phase between stress and strain yields solid-like and liquidlike properties, called the storage and loss moduli, respectively, which are then captured over a range of imposed frequencies. Rarely are the full spatial distributions of shear and normal stresses considered, primarily because they cannot be measured except at boundaries and the information was not deemed of particular interest in theoretical studies. Likewise, strain distributions throughout the layer were traditionally ignored except in a classical protocol of Ferry, Adler and Sawyer, based on snapshots of standing shear waves. Recent investigations of thin lung mucus layers exposed to oscillatory stress (breathing) and strain (coordinated cilia), however, suggest that the wide range of healthy conditions and environmental or disease assaults lead to conditions that are quite disparate from the "surface loading" and "gap loading" conditions that characterize classical rheometers. In this article, we extend our previous linear and nonlinear models of boundary stresses in controlled oscillatory strain to the entire layer. To illustrate non-intuitive heterogeneous responses, we characterize experimental conditions and material parameter ranges where the maximum stresses migrate into the channel interior.
\end{abstract}

\section{Introduction}

We first recall the formulation developed in [7,11,12], which is a generalization of the Ferry shear wave model [4-6] (also considered by Schrag et al. [15,16] for linear viscoelastic experiments) to finite depth layers and nonlinear constitutive laws. We briefly summarize the key elements from these references in order to describe the present focus on heterogeneous stress distributions in a viscoelastic layer being driven by oscillatory shear strain or stress. The equations of motion for an incompressible fluid are 1 ,

\footnotetext{
(C) 2010 IMACS. Published by Elsevier B.V. All rights reserved.

*Corresponding Author .

Publisher's Disclaimer: This is a PDF file of an unedited manuscript that has been accepted for publication. As a service to our customers we are providing this early version of the manuscript. The manuscript will undergo copyediting, typesetting, and review of the resulting proof before it is published in its final citable form. Please note that during the production process errors may be discovered which could affect the content, and all legal disclaimers that apply to the journal pertain.

${ }^{1}$ Throughout this paper, arrows will indicate column vectors, while bold symbols indicate tensors.
} 


$$
\begin{gathered}
\rho\left(\frac{\partial \vec{v}}{\partial t}+(\vec{v} \cdot \nabla) \vec{v}\right)=\nabla \cdot T \\
\nabla \cdot \vec{v}=0,
\end{gathered}
$$

where $\boldsymbol{T}$ is the total stress tensor, $\vec{v}$ is the fluid velocity, and $\rho$ is the fluid density. The total stress tensor is decomposed as $\boldsymbol{T}=-p I+\boldsymbol{\tau}$ where $p$ is the pressure [2] and $\boldsymbol{\tau}$ incorporates both the viscous stress and "extra stresses" arising from elastic properties of the material of interest.

Our baseline model is the upper-convected Maxwell (UCM) model (equations 3, 4 below with $a=0$ ), which possesses the nonlinearity common to all nonlinear differential constitutive models, and which reduces in the linear limit to a simple viscoelastic fluid with a viscous parameter $\left(\eta_{0}\right)$ and a relaxation time $\left(\lambda_{0}\right)$. We consider a single stress relaxation mode here, but any finite sum can be similarly studied. We will also consider the Giesekus model which adds a quadratic nonlinearity (in the stress tensor) to the UCM model,

$$
\lambda_{0} \stackrel{\nabla}{\tau}+\tau-a \tau: \tau=2 \eta_{0} D
$$

where the scalar parameter $a$ is the Giesekus mobility parameter, $\boldsymbol{D}$ is the rate-of-strain tensor, $D=1 / 2\left(\nabla \vec{v}+\nabla \overrightarrow{\boldsymbol{v}}^{T}\right)$, and the symbol over $\tau$ is the upper convected derivative [1,10],

$$
\stackrel{\nabla}{\tau}=\frac{\partial \tau}{\partial t}+(\vec{v} \cdot \nabla) \tau-\nabla \vec{v}^{T} \cdot \tau-\tau \cdot \nabla \vec{v}
$$

\section{Phasic Strain-Induced Stress Envelopes in the Upper Convected Maxwell Model}

We first recall from [11] the frequency-locked solutions for an arbitrary linear viscoelastic fluid and their relationship to the UCM equation and momentum equation for imposed oscillatory strain of frequency $\omega$ on a fluid in a finite depth channel. The standard Cartesian $x y z$ coordinate system will be used throughout the paper. The imposed boundary conditions on velocity are $V_{X}(0, t)=V_{0} \sin (\omega t), V_{X}(H, t)=0$, where $H$ is the height of the stationary boundary in the finite depth channel, and we assume $v_{y}=v_{z}=0$. We note the no-slip boundary condition applied to both surfaces is natural for most materials and our particular experimental applications, but that our analysis and numerics (and the implications thereof) are not limited to this constraint. Here, the moving surface displaces a maximum distance $A$ $=V_{0} / \omega$, and so the imposed bulk strain per oscillation can be defined as $\gamma_{0}=A / H$. For the general linear viscoelastic constitutive law, focusing on the shear stress component alone by the assumption of linearity,

$$
\tau_{x y}=\int_{0}^{t} G\left(t-t^{\prime}\right) \frac{\partial v_{x}}{\partial y}\left(y, t^{\prime}\right) d t^{\prime}
$$

the frequency-locked shear flow and stress for an arbitrary linear viscoelastic fluid are given by [12], 


$$
\begin{gathered}
v_{x}(y, t)=\operatorname{Im}\left(V_{0} e^{i w t} \frac{\sinh (\delta(H-y))}{\sinh (\delta H)}\right), \\
\tau_{x y}(y, t)=\operatorname{Im}\left(-\delta V_{0} \eta^{*} e^{i w t} \frac{\cosh (\delta(H-y))}{\sinh (\delta H)}\right) .
\end{gathered}
$$

Note that "Im" refers to the imaginary part of the complex expressions. The Fourier transform of the time-dependent modulus $G(t)$ is the frequency-dependent complex modulus $G^{*}(\omega)=G(\omega)+i G^{\prime}(\omega)$; characterizations of dynamic moduli either focus on the storage moduli $G(\omega)$ and loss moduli $G^{\prime}(\omega)$, or equivalently in terms of the complex viscosity $\eta^{*}=$ $\eta^{\prime}-i \eta^{\prime}$, where $G^{*}=i \omega \eta^{*}$. The key complex parameter in $(6,7)$ is $\delta=a+i \beta ; a$ and $\beta$ have the units of reciprocal length, and in the Ferry protocol for unidirectional shear waves they relate explicitly to reciprocals of the attenuation and oscillation lengthscales of shear wave snapshots [4-6]. In finite depth layers with counter-propagating waves, $a$ and $\beta$ lose explicit contact with shear wave snapshots, yet retain the same 1:1 relationship with $G^{\prime}$ and $G^{\prime}$ (or $\eta^{\prime \prime}$ and $\left.\eta^{\prime}\right)[12]$ :

$$
G^{\prime}=\rho \omega^{2} \frac{\beta^{2}-\alpha^{2}}{\left(\alpha^{2}+\beta^{2}\right)^{2}}, \quad G^{\prime \prime}=\rho \omega^{2} \frac{2 \alpha \beta}{\left(\alpha^{2}+\beta^{2}\right)^{2}} .
$$

The single mode Maxwell (equation 3) solution is recovered under the assumption that the linear viscoelastic kernel is a single exponential function $\left(G(t)=G_{0} e^{-t / \lambda_{0}}, \quad G_{0}=\eta_{0} / \lambda_{0}\right)$, whose Fourier transform $\left(G^{*}\right)$ is, of course, explicit,

$$
\begin{gathered}
\frac{G^{\prime \prime}}{\omega}=\eta^{\prime}=\frac{\eta_{0}}{1+\left(\omega \lambda_{0}\right)^{2}} \\
\frac{G^{\prime}}{\omega}=\eta^{\prime \prime}=\frac{\eta_{0} \omega \lambda_{0}}{1+\left(\omega \lambda_{0}\right)^{2}} ;
\end{gathered}
$$

$a$ and $\beta$ are likewise explicit:

$$
\alpha^{2}=\frac{\rho \omega}{2 \eta_{0}}\left(\sqrt{1+\omega^{2} \lambda_{0}^{2}}-\omega \lambda_{0}\right)
$$

In linear viscoelasticity, the normal stresses are presumed zero; however, the Lodge generalization of the linear tensor law easily provides an explicit formula for all stress components. The component $\tau_{y y}$ decays to zero at rate $1 / \lambda_{0}$, whereas $\tau_{x x}$ can be computed from a convolution integral,

$$
\tau_{x x}(y, t)=\int_{0}^{t} e^{\frac{t^{\prime}-t}{\lambda_{0}}} \frac{\partial}{\partial y} v_{x}\left(y, t^{\prime}\right) \tau_{x y}\left(y, t^{\prime}\right) d t^{\prime}
$$

In Lindley et. al. [11] boundary stress signals are analyzed from these formulas. Our aim here is to analyze the entire heterogeneous stress and flow/strain envelopes. By that, we mean that in any given experiment, every layer height $0 \leq y \leq H$ experiences an oscillatory strain and shear stress. We come back to the normal stress generated due to the upper 
convected derivative later. We focus now on the maximum over time of the velocity (equivalent to strain by a simple integration) and shear stress as a function of gap height $y$ in the layer. Together these data determine the flow and stress envelopes, the spatial curves which the oscillating flow and stress touch at their maximum over time. From $(6,7)$, for any linear viscoelastic constitutive law (5), the velocity and stress envelopes are given by,

$$
\begin{gathered}
v_{x}^{e n v}(y)=V_{0}\left|\frac{\sinh (\delta(H-y))}{\sinh (\delta H)}\right| \\
\tau_{x y}^{e n v}(y)=V_{0}\left|\delta \| \eta^{*}\right|\left|\frac{\cosh (\delta(H-y))}{\sinh (\delta H)}\right| .
\end{gathered}
$$

Recall the bulk strain is $\gamma_{0}=\boldsymbol{A} / \boldsymbol{H}$. Note that by normalizing $\boldsymbol{V}_{X}$ by $\boldsymbol{H}$, we get the normalized bulk strain rate envelope $\dot{\gamma}(y)$. We choose the following physical quantities to nondimensionalize this system: the reference lengthscale is the plate displacement amplitude $\boldsymbol{A}$, the reference timescale is proportional to the plate period $\omega^{-1}$, and the reference stress is the bulk viscous stress $\eta_{0} / \omega$. The following dimensionless parameters arise in the model equations:

- $\quad$ Reynolds number $\operatorname{Re}=\rho \omega A^{2} / \eta_{0}$.

- Deborah number $D e=\omega \lambda_{0}$.

- $\quad$ Bulk Shear Strain $\gamma_{0}=A / H$.

The lengthscale ratio $H / L$, where $L$ is the distance a shear or stress wave travels in one period of plate oscillation, plays prominently in our wave analysis from the full linear and nonlinear system of partial differential equations with either the Upper Convected Maxwell or Giesekus models [11]. Recall [11,12] that the "zero-stress" shear wave speed is:

$$
c_{0}=\sqrt{\frac{\eta_{0}}{\lambda \rho}} .
$$

Thus, a wave launched from the driven surface will traverse a distance $L$ during each oscillation,

$$
L=\frac{c_{0}}{\omega}
$$

since $1 / \omega$ is the period in seconds of the driven surface. The nondimensional length scale $H /$ $L$ can be written in terms of the above nondimensional variables,

$$
H / L=\frac{\sqrt{\operatorname{ReDe}}}{\gamma_{0}} .
$$

The wavespeed of a single mode depends on the relaxation timescale $\lambda_{0}$ and viscosity $\eta_{0}$, and it is straightforward to see that for biological fluids like lung mucus, even slow waves traverse a 100 micron thick sample in a fraction of a second, comprable to the cilia beat period. These basic observations lead us to explore stress distributions across the gap versus several dimensionless ratios. 
In Figure 1, we depict the envelopes for the stress and velocity in dimensional units (cgs) and show a few snapshots of shear waves and their corresponding envelopes at different points in time, for three disparate model fluids (where $G \gg G^{\prime}$ ' (a highly elastic fluid), $G \sim$ $G^{\prime}$ (viscoelastic fluid), and $G \ll G^{\prime}$ (nearly viscous fluid)). Recall that $\delta$ is a complex scalar, and from the behavior of hyperbolic functions along a ray in the complex plane, the non-monotone behavior of the figures becomes apparent. Here, the normal stress envelopes we show are obtained from numerical integration of (13). Note that, in Fig. 1, the peak stresses and velocities do not necessarily occur at the driven (or stationary) surface, particularly for a more elastic fluid. This is a natural consequence of bi-directional wave interference.

The peak shear stress will be felt at the point(s) $y$ which maximize $|\cosh (\delta(H-y))|$. Throughout this analysis, we will present the peak stress levels in three viscoelastic liquid regimes: strongly elastic, viscoelastic, and nearly viscous, following our previous work [11]. From the 1:1 relationship between $\left(G, G^{\prime}\right)$ and $(a, \beta)$ given in (8), the tuning of the material regime can also be controlled by the ratio $a / \beta \in(0,1)$, where $a / \beta \ll 1$ is the elastic limit, and $a / \beta=1$ is the viscous fluid limit. We now proceed with finding the peaks of the stress envelopes (that is, the gap height where the maximum stresses arise), and we begin by focusing on how the gap height of maximum stress scales with the layer height $H$. From the explicit formulas for the upper convected Maxwell model, the analogous scaling with other parameters follows. In biological layers such as mucus, the height $H$ modulates through drawing of water into and out of the airway. It is also natural to explore the frequency and displacement of coordinated cilia and the viscosity and relaxation time of the dominant relaxation mode.

In the context of waves traveling at finite speeds in an elastic solid we expect a resonance if the round trip distance for waves is precisely $2 H$. Thus, a resonance length $H_{r}$ can be identified as $H_{r}=L / 2$. What this means is that we observe a global peak of the stress envelope to occur at the stationary surface if the channel depth $H$ is less than $H_{r}$. If $H$ is increased to $H_{T}$ a precise harmonic will be reached, and the stress peak will occur at the bottom and top surface simultaneously. Increasing $H$ beyond this precise harmonic, we expect a second maxima of the stress envelope within the interior of the fluid domain at the point where the counter propagating waves meet, until the second multiple of the harmonic is reached, and a 3rd stress maxima will occur at the driven surface. In this idealized elastic material, as $H$ is increased further, the number of internal maxima will be precisely equal to the number of wavelengths of the shear wave resolved in the channel before reaching the stationary interface, i.e. the smallest multiple of $H_{r}$ which is less than $H$. We will now illustrate this by fixing $G_{0}=\eta_{0} / \lambda_{0}$, the density $\rho$, the plate displacement amplitude $A$, and the frequency $\omega$. Figure 2-a illustrates the locations of the peak stresses in a highly elastic fluid as a function of $H$.

As predicted, when the channel width is increased beyond $H_{r}$, and the first resonance length is exceeded, the global max of the stress shifts to the interior of the channel, while another local max occurs at the stationary interface. Note that in our discussion before, we considered an idealized elastic solid where there is no viscous loss, and so all of the stress peaks are global maxima, but the results in Fig. 2a are for a viscoelastic fluid (albeit a highly elastic one) and therefore the stress peak nearest the driven surface will be slightly larger than the peak at the stationary surface due to viscous loss. If we allow $H$ to become very large relative to $H_{T}$, eventually this structure will vanish as the viscous loss dominates the effect of counter-propagating waves. As we move out of the elastic limit, we expect the regularity of the peaks to break down and the locations to shift away from these resonance length scales. 
Figure $2 b-2 c$ demonstrate that the stress features persist for a generic viscoelastic fluid. Here, there is still a resonance condition, but the nonlinear viscoelastic dynamics evince spreading and irregularity of locations of the peak stress heights. This is precisely the behavior of boundary stress variations versus layer height (and all other parameters) explained in [11], generalized to the entire spatial distribution of shear and normal stresses. We expect that these structures rapidly vanish as the viscous limit is approached, which Figure 2d demonstrates.

We note that the critical points of the normal $\tau_{X X}$ and shear $\tau_{x y}$ stress components coincide. Further, the velocity peaks are merely offset from the stress peaks, as illustrated below in Fig. 3. This is to be expected as an illustration of the phase lag between strain and stress in a viscoelastic fluid.

Counter-propagating waves in a finite depth channel were considered decades ago by Schrag et al. $[15,16]$, who were interested in errors arising from two experimental limits which were routinely exploited in shear rheometers. The "gap loading" limit arises when the wavelength of the shear waves is much longer than the depth of the channel, and can arise from using a very viscous fluid, a low frequency deformation, or a very narrow gap $[15,16]$. Likewise, if the gap width is much larger than the length of the shear wave, in the "surface loading" limit, then counter-propagating waves attenuate suffciently rapidly (with nominal loss modulus) that they can be ignored, and viscoelastic characterization can be done on the freely propagating shear wave. The surface loading limit (which Ferry et al. $[6,15,16]$ imposed) for typical loss moduli is safely insured if the shear wavelength to gap width is 1:30 or smaller. Similar estimates of errors due to failure to resolve counter-propagating waves are given independent of these two limits in [12]. The analysis provided here, and its generalization to Giesekus models, applies to these special limits as well as all other parameter regimes. The heterogeneous phenomenon illustrated here, where maximum stress values arise in the interior of the shear gap, is never seen in the gap loading or surface loading limits. Indeed, the more generic phenomena of non-monotone stress distributions within the shear gap is not realized in these limits. As mentioned previously, we were led to explore the full parameter space (of driving conditions, layer depth and material properties) in order to understand phasic shear driving conditions in thin mucus layers lining lung pathways.

For the purposes of this article, we focus on the heterogeneous behavior of shear and normal stress envelopes versus two dimensionless lengthscales: $a / \beta \in(0,1)$, the ratio of unidirectional shear wave oscillation to attenuation length scales; and the ratio $H / L \in(0$, $\infty$ ), of the gap height to the distance $L$ traveled by a propagating shear wave in one period of oscillation of the driven plate. The gap loading limit corresponds to $H / L \ll 1$ and the surface loading limit corresponds to $H / L \gg 1$, whereas we are interested in the entire twoparameter strip of $a / \beta$ in $(0,1)$ and $H / L$ in $(0, \infty)$. To focus on a particular behavior, we are interested in the domains in this strip where the stress envelopes have an internal maximum.

Table 1 and Figure 4 give the 2-parameter domains for each relaxation mode of a viscoelastic fluid where the gap loading and surface loading limits apply, and where internal stress maxima occur. Figure 4a shows a spline fit over the largest intervals where the phenomenon is present, while Fig. $4 \mathrm{~b}$ shows the precise parameter regimes where the internal stress maxima phenomenon occurs.

\section{Higher Order Nonlinearity}

For the particular nonlinearity beyond the upper convective derivative that is special to the Giesekus model, we explore the above phenomena as the mobility parameter is varied from zero (the UCM model) i.e. equation (3) with $a \neq 0$. We demonstrate that the non-monotone 
character of the stress envelopes discussed above are modified but the essential features persist. To explore this nonlinear model, we turn to a numerical approximation [12]. Because it is computationally expensive to compute the shear and normal stress envelopes many times for many heights even for one fixed parameter set, much less across a multiparameter space, we show only the results of varying the gap height for selected values of the Giesekus parameter a, with all other parameters held fixed. Similar non-monotone envelopes and variations with respect to parameters exist with respect to frequency and viscoelastic parameter sweeps. The cost is due to the fact that formulas for the shear velocity, shear and normal stresses have to be replaced in the Giesekus model by solution of a system of nonlinear partial differential equations for their space-time dependence. We refer to our previous papers for the detailed system of equations and numerical methods.

Figure 5 demonstrates the effect of the mobility parameter on the flow and stress envelopes. Here the mobility parameter is set at $a=.65$, and the other fluid parameters are the same as Model Fluid 2. The higher the value of the mobility parameter, the more shear thinning one will observe, and generally higher imposed bulk shear strains on the driven surface will cause more shear thinning within the channel. Here the same numerical simulation is run for both the UCM and Giesekus fluids, and the final time is set at $50 \times \lambda_{0}$ to ensure that transient effects have passed. The velocity and stress profiles for both fluids are given in Fig $5 a-5 c$. The structure of the envelopes perturbs under the Giesekus nonlinearity, indicating a departure from the sharp results obtained in the UCM limit. The key non-dimensional parameters governing the onset of shear thinning, and the deviation from the linear viscoelastic and UCM models are the mobility parameter $a$ and the bulk shear strain $\gamma_{0}=A$ / $H$. For larger values of $a$ or $\gamma_{0}$, we observe a walk off from the expected locations of the stress envelopes catalogued above. Fig. 6 shows the location of the peak stresses for both a sample UCM and Giesekus parameter sets.

\section{Conclusions}

We have explored the response of a viscoelastic layer in oscillatory shear, focusing on the spatial distribution of shear and normal stresses in the layer. We use the upper convected Maxwell model and the Giesekus model to explore nonlinear contributions to an underlying linear viscoelastic stress response structure in the layer. The linear stress response structure is exactly solvable in closed form, and the shear stress formula explicitly reveals nonmonotone distributions of stress in the shear gap, as well as parameter regimes where the maximum shear stress arises in the interior of the layer. The upper convective derivative, for the frequency-locked response, has the identical velocity and shear stress distributions, coupled with an explicit normal stress distribution suppressed by the assumption of linearity. Finally, the Giesekus quadratic stress nonlinearity is shown by numerical simulations of a coupled system of four quasi-linear partial differential equations to modify the analytical features of the UCM model, while preserving the fundamental features of non-monotonicity of stress envelopes in the shear gap and the possibility of maximum stresses arising in the layer interior. Such behavior implies that localized shear thinning of viscoelastic layers in oscillatory strain driving conditions can arise anywhere in the layer, and in particular not always at the driven interface. The implications of these phenomena for transport of mucus layers in lung pathways is a subject of future interest.

\section{Acknowledgments}

M. Gregory Forest's research is supported through the NSF DMS-0502266 and DMS-0908423, the NIH R01 HL077546-01A2, and the Department of Energy DE-SC0001914. Sorin Mitran's research is supported through the NIH RO1-HL077546-5401A2 and also through the Department of Energy DE-SC0001914. David B. Hill's work is supported through the Virtual Lung Project NIH grant Ro1-HL077546-03A2 and through the Cystic Fibrosis 
Foundation HILL08I0. The authors would also like to acknowledge the astute feedback of an anonymous reviewer who helped put these results into their most useful and general context.

\section{References}

[1]. Bird, R.; Curtis, C.; Armstrong, R.; Hassenger, O. Dynamics of Polymer Fluids. Vol. 1 \& 2. Wiley; New York: 1987.

[2]. Dealy JM. Misuse of the term Pressure in Rheology. Rheology Bulletin. 2008; 77:11-13.

[3]. Ewoldt, RH.; Hosoi, AE.; McKinley, GH. Rheological Fingerprinting of Complex Fluids using Large Amplitude Oscillatory Shear (LAOS) Flows. Annual Transactions of the Nordic Society of Rheology; Nordic Rheology Conference; Stavanger, Norway. 13-15 June 2007; p. 3-8.

[4]. Ferry JD. Studies of the Mechanical Properties of Substances of High Molecular Weight I. A Photoelastic Method for Study of Transverse Vibrations in Gels. Rev. Sci. Inst. 1941; 12:79-82.

[5]. Ferry JD. Behavior of Concentrated Polymer Solutions under Periodic Stresses. J. Polym. Sci. 1947; 2:593-611.

[6]. Ferry JD, Adler FT, Sawyer WM. Propagation of Transverse Waves in Viscoelastic Media. J. Appl. Phys. 1949; 20:1036-1041.

[7]. Hill DB, Lindley B, Forest MG, Mitran SM, Superfine R. Experimental and modeling protocols from a micro-parallel plate rheometer. 2009 UNC Preprint.

[8]. Jeyaseelan RS, Giacomin AJ. Network theory for polymer solutions in large amplitude oscillatory shear. J. Non-Newtonian Fluid Mech. 2007; 148:24-32.

[9]. Keunings R, Atalik K. On the Occurrence of Even Harmonics in the Shear Stress Response of Viscoelastic Fluids in Large Amplitude Oscillatory Shear. J. Non-Newtonian Fluid Mech. 2004; 122:107-116.

[10]. Larson, RG. Constitutive Equations for Polymer Melts and Solutions. Butterworths; Guilford UK: 1988.

[11]. Lindley B, Howell E, Smith BD, Forest MG, Rubenstein GJ, Mitran SM, hill DB, Superfine R. Stress Communication and Filtering of Viscoelastic Layers in Oscillatory Shear. J. NonNewtonian Fluid Mech. 2009; 156:112-120.

[12]. Mitran SM, Forest MG, Yao L, Lindley B, Hill D. Extenstions of the Ferry Shear Wave Model for Active Linear and Nonlinear Microrheology. J. Non-Newtonian Fluid Mech. 2008; 154:120135.

[13]. Preziosi L. On an Invariance Property of the Solution to Stokes First Problem for Viscoelastic Fluids. J. Non-Newtonian Fluid Mech. 1989; 33(2):225-228.

[14]. Preziosi L, Joseph DD. Stokes' First Problem for Viscoelastic Fluids. J. Non-Newtonian Fluid Mech. 1987; 25(3):239-259.

[15]. Schrag JL, Guess JF, Thurston GB. Shear Wave Interference Observed By Optical Birefringence Induced In A Viscoelastic Liquid. J. Appl. Phys. 1965; 36(1996)

[16]. Schrag JL. Deviation of Velocity Gradient Profiles from the "Gap Loading" and "Surface Loading" Limits in Dynamic Simple Shear Experiments. Trans. Soc. Rheo. 1977; 21(3):399-414.

[17]. Tarran R, Button B, Picher M, Paradiso AM, Ribeiro CM, Lazarowski ER, Zhang L, Collins PL, Pickles RJ, Fredberg JJ, Boucher RC. Normal and Cystic Fibrosis Airway Surface Liquid Homeostasis: The Effects of Phasic Shear Stress and Viral Infections. J. Biol. Chem. 2005; 280(42):35751-9. [PubMed: 16087672]

[18]. Tee TT, Dealy JM. Nonlinear Viscoelasticity of Polymer Melts. Trans. Soc. Rheol. 1975; 19:595-615. 


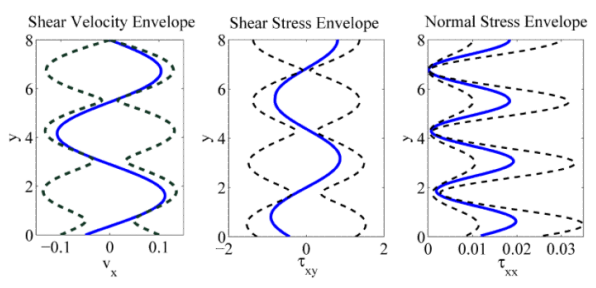

(a) Shear wave, shear stress, and normal stress snapshots and envelopes for a highly elastic fluid.

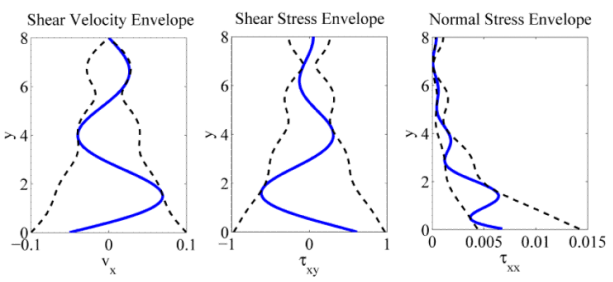

(b) Shear wave, shear stress, and normal stress snapshots and envelopes for a model viscoelastic fluid.

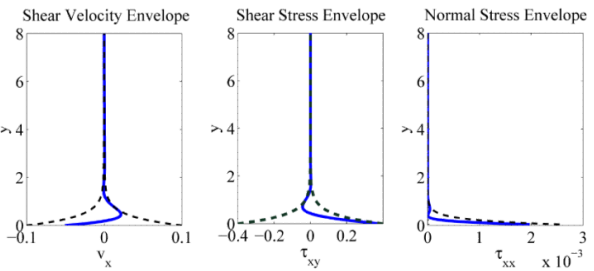

(c) Shear wave, shear stress, and normal stress snapshots and envelopes for a weakly elastic fluid.

Fig. 1.

Shear wave, shear stress, and normal stress snapshots for 3 model fluids a) highly elastic fluid b) generic viscoelastic fluid c) weakly elastic fluid. Each experiment is done under a bulk strain of $\gamma_{0}=.001$ (note $A=V_{0} / \omega$ ) and the parameter $H / L$ is 1.612 in each case. The dotted lines here represent the envelopes (bounds) on either the velocity or stress. The model fluids used here are 1) $\eta_{0}=100 \mathrm{gcm}^{-1} \mathrm{~s}^{-1}, \lambda_{0}=1, R e=8.077 \times 10^{-6}, D e=12.57$; 2) $\eta_{0}=$ $20 \mathrm{gcm}^{-1} \mathrm{~s}^{-1}, \lambda_{0}=.2 \mathrm{~s}, R e=4.039 \times 10^{-5}, D e=2.541$ and 3) $\eta_{0}=1 \mathrm{gcm}^{-} \mathrm{s}^{-1}, \lambda_{0}=.01 \mathrm{~s}, R e=$ $8.077 \times 10^{-4}, D e=.1257$ with density $\rho=1.015 \mathrm{gcm}^{-3}$, and the axes values are in cgs units. The frequency is fixed at $2 \mathrm{~Hz}$. 

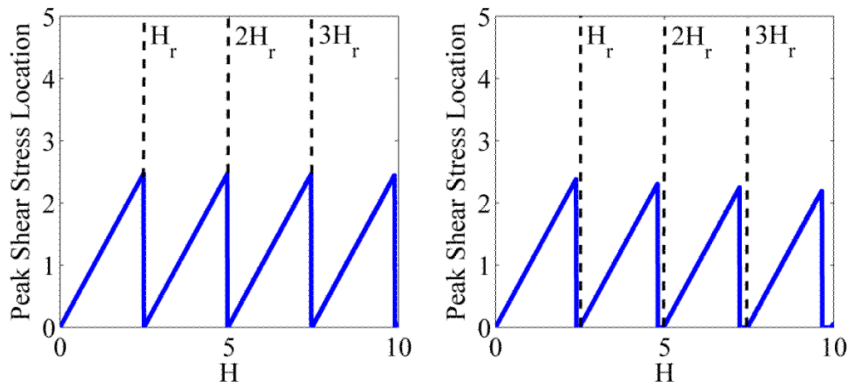

(a) $R e=.0128, D e=125.7$,(b) $R e=.1275, D e=12.57$, $\alpha / \beta=3.979 \times 10^{-3}$ $\alpha / \beta=3.973 \times 10^{-2}$
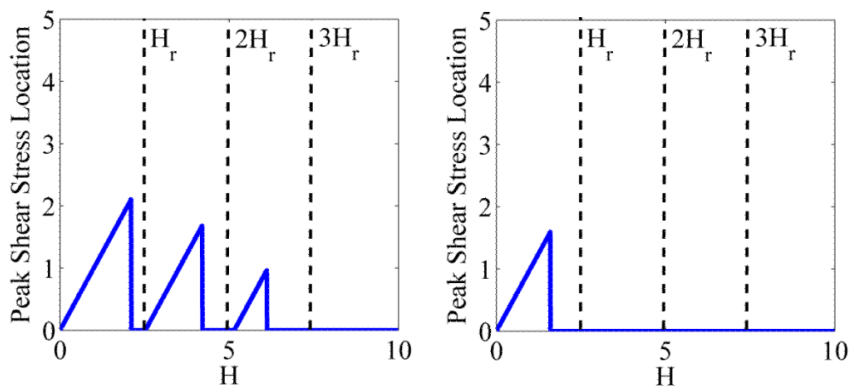

(c) $R e=.5102, D e=3.142$,(d) $R e=1.275, D e=1.257$, $\alpha / \beta=.1553$

$\alpha / \beta=.3493$

Fig. 2.

The spatial locations of the peak stress values in non-dimensional units of length $(H / A)$, where $H$ is the layer thickness and $A$ is the deformation amplitude. Here $A$ is fixed while $H$ is varied, and thus the non-dimensional bulk shear strain $\gamma_{0}=A / H$ varies over the range $.1 \leq$ $\gamma_{0} \leq \infty$. The figure shows where the maximum of the stress envelope occurs spatially (vertical axis) at any fixed gap width (horizontal axis). The dotted lines are at the elastic resonance condition $H_{r}=2.4815$ and the higher harmonics (i.e. multiples of $H_{r}$ ). In figures a-d, we move from the highly elastic to the weakly elastic limit in order. Note that in all cases the driving parameters and $G_{0}=\eta_{0} / \lambda_{0}$ are identical, which fixes $c_{0}=9.926 \mathrm{~cm} / \mathrm{s}$ (equation (16)) and the elastic resonance height $H_{r}$. 

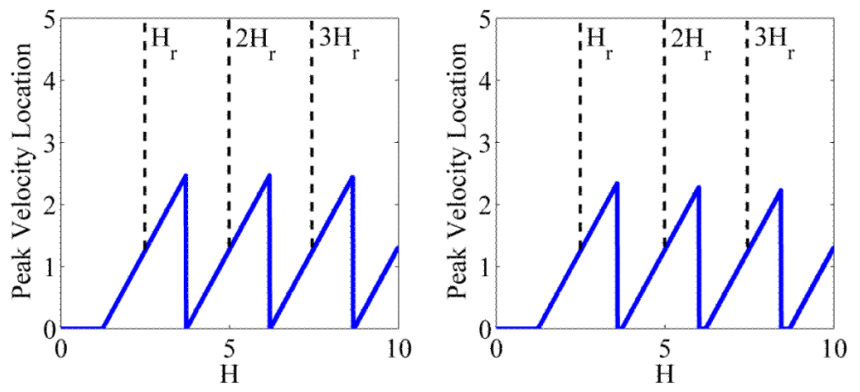

(a) $R e=.0128, D e=125.7$,(b) $R e=.1275, D e=12.57$, $\alpha / \beta=3.979 \times 10^{-3}$

$\alpha / \beta=3.973 \times 10^{-2}$
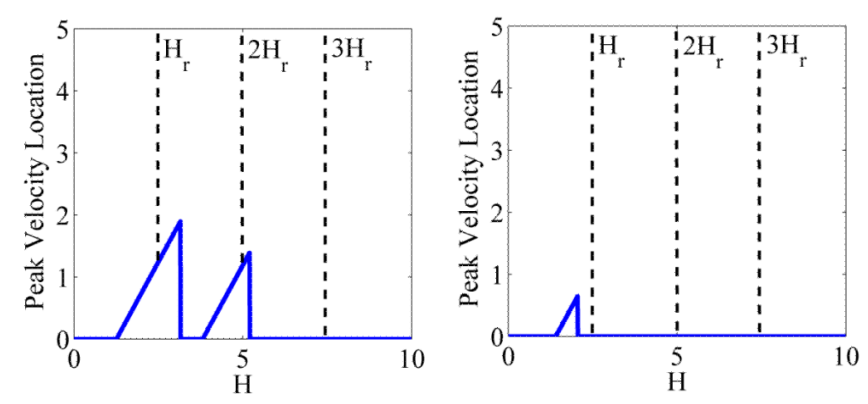
(c) $R e=.5102, D e=3.142$,

$\alpha / \beta=.1553$

(d) $R e=1.27$
$\alpha / \beta=.3493$

Fig. 3.

The spatial locations of the peak flow rates. These are the flow envelopes associated with the stress envelopes in Figure 2. 


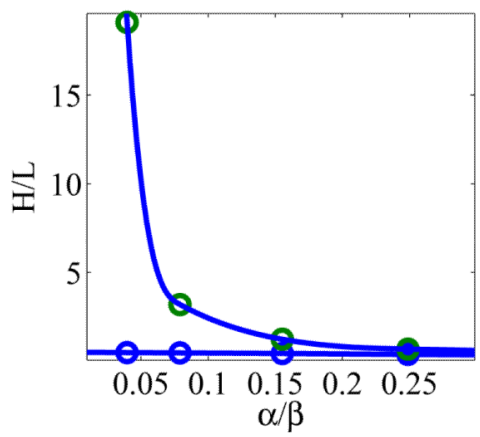

(a)

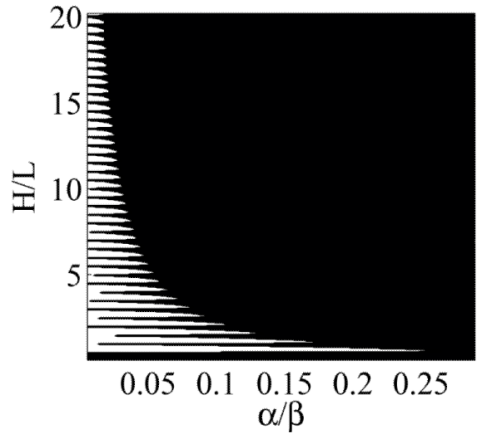

(b)

Fig. 4.

Figures 4 a) and b) are a graphical representation of the region where the internal maximum stress phenomenon occurs. Fig. 4a is a spline interpolation of the data points in Table 1, and the region between the two lines is a reasonable approximation of where the phenomenon occurs. Fig. 4b illustrates, precisely, where the stress phenomenon occurs (the white regions), and is derived from conditions on equation (15). In both experiments, $G_{0}, \rho$ and $\omega$ (and consequently the wave propagation speed) are held constant, while material parameters and channel width are systematically varied. 


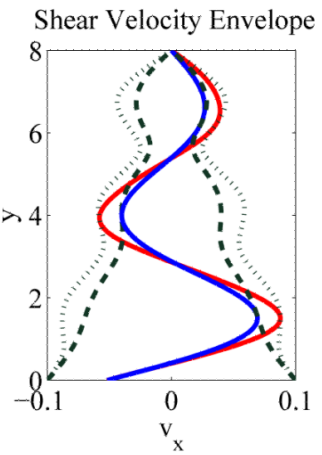

(a)

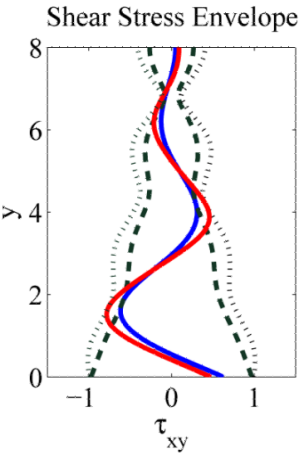

(b)

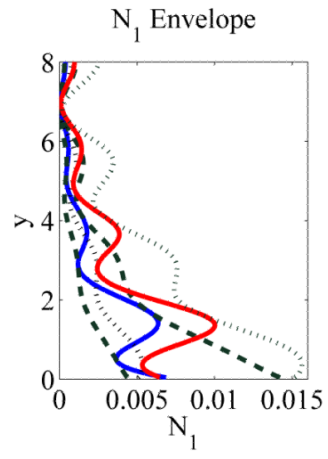

(c)

Fig. 5.

The effect of the mobility parameter on the spatial distributions of velocity/stress. Here a model fluid is considered with $\eta_{0}=20 \mathrm{gcm}^{-1} \mathrm{~s}^{-1} \lambda_{0}=.2 \mathrm{~s}$ and $a=0$ or $a=.65$. The nondimensional bulk shear strain $A / H$ is fixed to be .001 in both experiments $(\operatorname{Re}=4.039 \times$ $10^{-5}$ and $D e=2.541$ ). The Giesekus model is in red, with the associated envelopes shown as dots, while the UCM is shown in blue and the associated envelopes are dashes. The $N_{1}$ data is the first normal stress difference for the two models. 


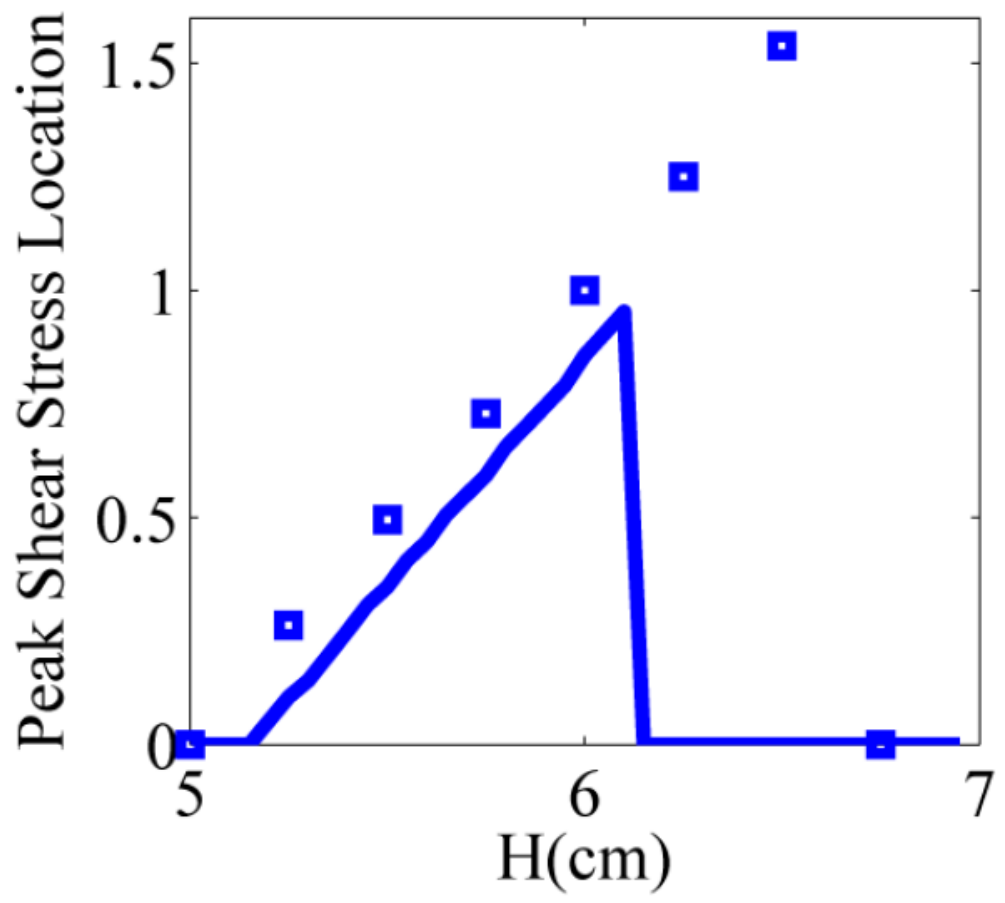

Fig. 6.

The location of the peak stresses for the UCM and Giesekus models as a function of different gap heights for the same parameters as in Figure 2c, with $a=.65$ for the Giesekus model. The Giesekus data is represented by square markers, while the UCM data is given by the solid line. 


\section{Table 1}

The occurrence of the first and last internal stress maximum over a range of fluid values, from highly elastic to mostly viscous, in channels of varying width at a fixed deformation amplitude. The values given are scaled by $H_{r}$, the predicted resonance height for a highly elastic fluid. The fluid values from these simulations cover the same range of values used in Fig. 2 and 3 i.e. $0128 \leq R e \leq 1.275,1.257 \leq D e \leq 125.7$ while the bulk strain is varied $.0019 \leq \gamma_{0} \leq \infty$

\begin{tabular}{|c|c|c|}
\hline $\boldsymbol{a} / \boldsymbol{\beta}$ & First Internal Max $/ \boldsymbol{H}_{\boldsymbol{r}}$ & Last Internal Max/ $\boldsymbol{H}_{\boldsymbol{r}}$ \\
\hline .0040 & .9999 & 208.0 \\
\hline .0397 & .9631 & 38.18 \\
\hline .0791 & .9228 & 6.379 \\
\hline .1553 & .8543 & 2.4663 \\
\hline .2488 & .7536 & 1.354 \\
\hline .3493 & .6488 & na \\
\hline
\end{tabular}

